\title{
Reversible Information Flow across the Medial Temporal Lobe: The Hippocampus Links Cortical Modules during Memory Retrieval
}

\author{
Bernhard P. Staresina, Elisa Cooper, and Richard N. Henson \\ Medical Research Council Cognition and Brain Sciences Unit, Cambridge CB2 7EF, United Kingdom
}

A simple cue can be sufficient to elicit vivid recollection of a past episode. Theoretical models suggest that upon perceiving such a cue, disparate episodic elements held in neocortex are retrieved through hippocampal pattern completion. We tested this fundamental assumption by applying functional magnetic resonance imaging (fMRI) while objects or scenes were used to cue participants' recall of previously paired scenes or objects, respectively. We first demonstrate functional segregation within the medial temporal lobe (MTL), showing domain specificity in perirhinal and parahippocampal cortices (for object-processing vs scene-processing, respectively), but domain generality in the hippocampus (retrieval of both stimulus types). Critically, using fMRI latency analysis and dynamic causal modeling, we go on to demonstrate functional integration between these MTL regions during successful memory retrieval, with reversible signal flow from the cue region to the target region via the hippocampus. This supports the claim that the human hippocampus provides the vital associative link that integrates information held in different parts of cortex.

\section{Introduction}

How does the sight of a vase on one's desk rekindle a memory of the market stall in which it was purchased? Theoretical accounts and computational models posit that after initial binding, a partial retrieval cue will elicit a pattern completion process that reinstates the constituents of the original experience. Importantly, this process is thought to be accomplished by the hippocampus, a key region of the medial temporal lobe (MTL) memory system (Marr, 1971; Teyler and DiScenna, 1986; Treves and Rolls, 1994; Norman and O'Reilly, 2003; Teyler and Rudy, 2007). For instance, according to the "hippocampal memory indexing theory," the actual contents of a memory representation are held in cortex, and these cortical regions are dynamically linked by the hippocampus during successful memory retrieval (Teyler and DiScenna, 1986; Teyler and Rudy, 2007). Although neuropsychological studies have shown clear evidence for the destructive effects of hippocampal damage on episodic memory (Squire, 1992; Yonelinas et al., 2002; Mayes et al., 2004, 2007; Squire et al., 2004; Vann et al., 2009), these findings cannot answer how the hippocampus dynamically interacts with cortical modules during intact episodic memory retrieval.

However, understanding the MTL's network dynamics (or "functional integration") during memory retrieval first requires

\footnotetext{
Received May 11, 2013; revised June 26, 2013; accepted July 22, 2013.

Author contributions: B.P.S. and R.N.H. designed research; B.P.S. and E.C. performed research; B.P.S. and R.N.H. analyzed data; B.P.S. and R.N.H. wrote the paper.

This work was supported by a Sir Henry Wellcome Postdoctoral Fellowship to B.P.S. and the UK Medical Research Council Program MC_A060_5PR10 to R.N.H. We thank Mike Anderson for helpful discussion.

The authors declare no competing financial interests.

Correspondence should be addressed to Dr. Bernhard Staresina, MRC Cognition and Brain Sciences Unit, 15 Chaucer Road, Cambridge CB2 7EF, UK. E-mail: bernhard.staresina@mrc-cbu.cam.ac.uk.

DOI:10.1523/JNEUROSCI.1987-13.2013

Copyright $\odot 2013$ the authors $\quad 0270-6474 / 13 / 3314184-09 \$ 15.00 / 0$
}

understanding the separate contributions (or "functional segregation") among its subregions. While there is broad consensus about the role of the hippocampus (HIPP) in associative encoding and retrieval (for reviews, see Squire et al., 2004; Davachi, 2006; Eichenbaum et al., 2007; Mayes et al., 2007), controversy still surrounds the putative roles of perirhinal ( $\mathrm{PrC}$ ) and parahippocampal cortex $(\mathrm{PhC})$ (comprising the anterior and posterior portions of the parahippocampal gyrus, respectively). While "process-based" accounts emphasize differential contributions of $\mathrm{PrC}$ and $\mathrm{PhC}$ to familiarity-based versus recollection-based recognition (Aggleton and Brown, 1999; Diana et al., 2007; Eichenbaum et al., 2007; Mayes et al., 2007), more recent "domain-based" accounts, building largely on neuroanatomical data (Suzuki and Amaral, 1994; Burwell and Amaral, 1998; Lavenex and Amaral, 2000), emphasize different stimulus properties processed by $\mathrm{PrC}$ and $\mathrm{PhC}$ (Davachi, 2006; Graham et al., 2010; Wixted and Squire, 2011). A key prediction of such domain-based accounts is that both $\mathrm{PrC}$ and $\mathrm{PhC}$ may contribute to recollection, but differentially so as a function of the stimulus material used to define successful memory performance.

In the current study, we used a novel object-scene cued recall paradigm (Fig. 1) to first assess whether PrC and $\mathrm{PhC}$ differentially process object- and scene-related information, respectively (consistent with domain-based accounts). Importantly, we then set out to reveal how these stimulus-specific contributions might be dynamically integrated during object-scene recall, and whether there is indeed a role of HIPP in linking the episodic elements held in cortex.

\section{Materials and Methods}

Participants. Twenty (11 female) right-handed native English speakers with normal or corrected-to-normal vision participated in the experiment (mean age: 25 years, range: $22-32$ ). Informed consent was obtained 


\section{Encoding}
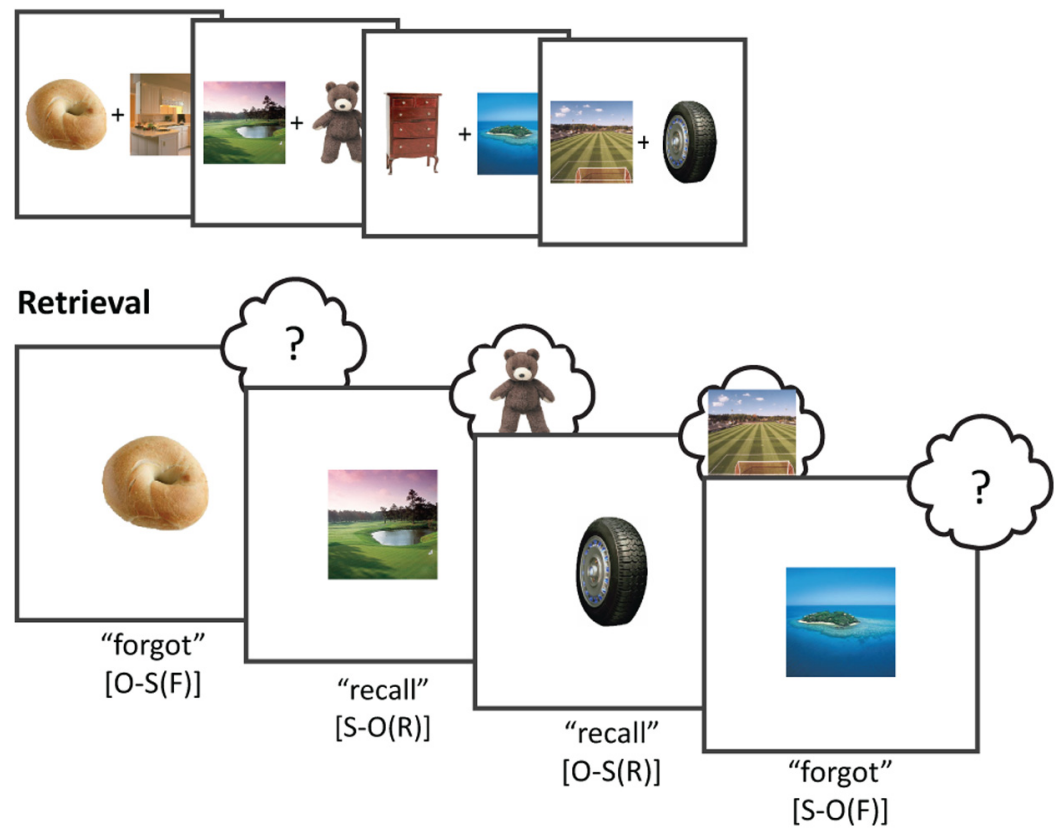

Figure 1. Experimental paradigm. During encoding, participants were presented with pairs of trial-unique object and scene images. During retrieval, participants were either cued with an object or with a scene, and indicated whether they could recall the corresponding target. $0-\mathrm{S}(\mathrm{R})$ : object cue, scene target recalled; $0-\mathrm{S}(\mathrm{F})$ : object cue, scene target forgotten; $\mathrm{S}-\mathrm{O}(\mathrm{R})$ : scene cue, object target recalled; $\mathrm{S}-\mathrm{O}(\mathrm{F})$ : scene cue, object target forgotten.

in a manner approved by a local Psychological Research Committee and participants were paid for their participation.

Experimental design. The stimulus material consisted of 384 color pictures (Konkle et al., 2010), half of which (192) depicted objects and half of which depicted scenes ( 16 additional pictures were used for practice). For each of the two stimulus categories (objects, scenes), there were two similar exemplars for each of 96 subcategories (e.g., a glass of red wine and a glass of white wine for the object subcategory "wine glass" or a scene with a volcano emitting lava and scene with a volcano emitting an ash cloud for the scene subcategory "volcano"). As detailed below, the two exemplars per subcategory were used to enforce attention to eventspecific details during encoding and retrieval. The stimulus material was counterbalanced so that half of the participants were presented with set 1 of subcategory exemplars during the first half of the experiment and with set 2 during the second half (and vice versa for the other half of the participants). Thus, no two exemplars of a given subcategory were presented within the same encoding-retrieval cycle.

The experiment consisted of six runs, with each run containing three blocks: an encoding block, a delay block, and a retrieval block (Fig. 1). Scanning was performed continuously across the three blocks, with short unscanned breaks between runs. Only retrieval data are reported here. The experiment was presented via the Psychophysics Toolbox (Brainard, 1997) implemented in MATLAB. During each encoding block, participants were presented with 32 unique object-scene pairs. The pairing of objects and scenes was randomized across participants. Object and scene pictures were each presented in a $250 \times 250$ pixels frame placed to the left and right of the screen center. During half of the trials (16, randomly selected), the object appeared to the left of the screen center and the scene to the right, with the reverse order during the other half of the trials. The trial duration was $4 \mathrm{~s}$, and for the last $0.5 \mathrm{~s}$ the picture pair was replaced with a fixation cross (responses were still recorded), alerting participants that another trial would appear shortly. The encoding task was to indicate via button press whether the given object-scene pair is plausible or implausible, i.e., likely to appear in real life or nature (Staresina and Davachi, 2006). "Plausible" responses were given with the index finger and "implausible" responses with the middle finger. Across participants, use of left versus right hand was counterbalanced (but the finger assignment was held constant). Object-scene encoding trials were intermixed with an active baseline condition (Stark and Squire, 2001). Here, random numbers between 0 and 100 were shown, and participants pressed the index finger key for even numbers and the middle finger key for odd numbers. As soon as a response was given, another random number was shown. The response time for each number was self-paced and participants were encouraged to perform this task as fast as possible without sacrificing accuracy. Each encoding block lasted $\sim 3$ min.

After the last encoding trial, participants saw a transition screen for $16 \mathrm{~s}$, alerting them to the upcoming delay block. During the delay block, participants again performed the odd/even numbers task described above for $2 \mathrm{~min}$. Odd/ even response accuracy was reported to participants on the computer screen following the completion of the task to encourage accuracy.

At the end of the delay block, another $16 \mathrm{~s}$ transition screen alerted participants to the upcoming retrieval block. Each retrieval block consisted of 32 trials, each trial lasting $6 \mathrm{~s}$. For a given trial, participants saw either the object or the scene of a given object-scene pair from the previous encoding block and were asked to indicate whether they remembered the corresponding paired associate ("recall"; index finger) or not ("forgot"; middle finger). Half of the cues (16) were object pictures, the other half scene pictures. Across the 32 retrieval trials, each cue type (object cue or scene cue) was presented in mini-blocks of eight consecutive trials (A-B-A-B), with a random assignment of object and scene cue trials to $A$ and $B$ in each run. Participants received the following instructions regarding "recall" responses: "Remembering the associate means that you could describe it in such a way that another person who has not seen the stimuli can pick the correct stimulus based on your description. Keep in mind that there are multiple exemplars per category, so your description has to be as detailed as possible." As mentioned above, there were only two exemplars per subcategory. To ensure that participants gave "recall" responses when they indeed recalled the correct paired associate, we asked them to verbally describe the target after $\sim 10 \%$ of the "recall" responses. In particular, three catch trials per block were randomly determined beforehand (e.g., trial 5, 14, and 32 for block 1 ). If the participant did not give a recall response on a designated catch trial, the next trial on which a recall response was given served as a catch trial. This means that for some blocks, there were $<3$ catch trials (e.g., if the participant indicated forgot on trial 32 (the last trial in a block) in the example above so that no alternative catch trial could be chosen). Catch trials started after the $6 \mathrm{~s}$ trial period, showing a $2 \mathrm{~s}$ warning screen ("prepare to describe the associated image...") followed by a $10 \mathrm{~s}$ period during which a verbal response was recorded ("please describe the associated image"), followed again by a $6 \mathrm{~s}$ fade-out screen ("prepare to continue with the experiment"). For scoring purposes, verbal responses were classified as accurate if they encompassed the target image's basic level label as well as some characteristic feature (e.g., "a glass of red wine" in the example above). As during the encoding block, retrieval trials were intermixed with odd/even number baseline trials. The retrieval block lasted $\sim 6 \mathrm{~min}$.

For the imaging analysis, the four conditions of interest were as follows: (1) object cue, target scene recalled (O-S(R)), (2) object cue, target scene forgotten $(\mathrm{O}-\mathrm{S}(\mathrm{F}))$, (3) scene cue, object target recalled (S-O $(\mathrm{R})$ ), and (4) scene cue, object target forgotten $(\mathrm{S}-\mathrm{O}(\mathrm{F}))$.

Magnetic resonance imaging scanning details. Scanning was performed on a $3 \mathrm{~T}$ Siemens Tim Trio magnetic resonance imaging (MRI) system using a 32-channel whole-head coil. Functional data were acquired using a gradient-echo, echo-planar pulse sequence $(\mathrm{TR}=1000 \mathrm{~ms}, \mathrm{TE}=30$ ms, 16 horizontal slices oriented parallel to the hippocampal axis, de- 
scending slice acquisition, $3 \times 3 \times 3 \mathrm{~mm}$ voxel size, $0.75 \mathrm{~mm}$ interslice gap, 702 volume acquisitions per run). The first 7 volumes of each run were discarded to allow for magnetic field stabilization. High-resolution $(1 \times 1 \times 1 \mathrm{~mm})$ T1-weighted (MP-RAGE) images were collected for anatomical visualization. Foam padding was used to minimize head motion. Visual stimuli were projected onto a screen that was viewed through a mirror, and responses were collected with magnet-compatible button boxes placed under the participant's hands.

The active baseline task (odd/even-task; Stark and Squire, 2001) comprised a fourth of the total scanning time. The sequence of encoding/ retrieval trials and the variable number of baseline trials was pseudorandom and optimized for rapid event-related functional MRI (fMRI; using the "optseq" algorithm; Dale, 1999).

fMRI analysis. Data were analyzed using SPM8 (http://www.fil.ion.ucl. ac.uk/spm/). During preprocessing, images were corrected for differences in slice acquisition timing, followed by motion correction across all runs. Neural activity for the conditions of interest $(\mathrm{O}-\mathrm{S}(\mathrm{R}), \mathrm{O}-\mathrm{S}(\mathrm{F})$, $\mathrm{S}-\mathrm{O}(\mathrm{R}), \mathrm{S}-\mathrm{O}(\mathrm{F})$ ) was modeled as an impulse (delta function) in a design matrix that concatenated all retrieval blocks and included nuisance regressors for invalid trials, head movement, low-frequency scanner drift, and run means. Additionally, the $10 \mathrm{~s}$ overt speech plus the surrounding $2 \mathrm{~s}$ fade-in and $6 \mathrm{~s}$ fade-out periods of catch trials were modeled as user-specified nuisance regressors (using unconvolved stick functions for each volume). For the conventional general linear model (GLM) analysis, condition onsets were convolved using a single, canonical hemodynamic response function (HRF), as provided in SPM8. The resulting $\beta$-parameter estimates were then averaged across voxels within each region of interest (ROI; see below) in the participant's native space, and the resulting values were used in repeated-measures ANOVAs and $t$ tests. For ANOVA factors with more than one numerator degree of freedom (df), we used a Greenhouse-Geisser df-correction for nonsphericity of the error.

Extraction of time-resolved blood oxygenation level-dependent (BOLD) data was based on the same design matrix, but condition responses were modeled via a finite impulse response (FIR) basis set (rather than the canonical HRF), with 20 bins and a $1 \mathrm{~s}$ bin-width equal to the TR (and converted to percentage signal change via the MarsBaR toolbox; Brett et al., 2002). We focused on the evoked BOLD response, corresponding to the first 11 bins, or $0.5-11.5 \mathrm{~s}$ (given that data were aligned to the middle slice acquired). These FIR parameter estimates were averaged across voxels within each ROI in the participant's native space.

We analyzed the data in PrC, PhC, and HIPP using hand-drawn, participant-specific ROIs, based on the individual structural image. Anatomical demarcation was done according to Insausti et al. (1998) and Pruessner et al. (2002). As there were no hemispheric differences (data not shown), we combined left and right hemisphere ROIs. Specifically, data were separately extracted for left and right hemisphere ROIs and collapsed before entering analyses. Note that no spatial smoothing was performed on the data, ensuring that there was minimal signal overlap between the regions.

Nonlinear HRF fitting. To estimate the BOLD onset latency, we fit the model:

$$
\mathbf{y}=g\left(\mathbf{t}, p_{1}, p_{2}, p_{3}\right)+\mathbf{e},
$$

where $y$ is the vector of the above 11 FIR parameter estimates across time

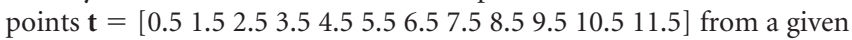
ROI and condition of a given participant, $\mathbf{e}$ is random error, and $g$ is a nonlinear function with parameters $p_{1-3}$. Given the positively skewed nature of the BOLD response, we defined $g$ as a Gamma function:

$$
g\left(t, p_{1}, p_{2}, p_{3}\right)=p_{1} \times G\left(t-p_{2}, 6 / p_{3}, d t / p_{3}\right),
$$

where $p_{1}$ is a scaling parameter (amplitude), $p_{2}$ is the onset latency, and $p_{3}$ is a dispersion factor that affects the shape and scale of a gamma probability density function $(G)$ over time $t$, relative to a peak of $6 \mathrm{~s}$ when sampled every $d t=1 \mathrm{~s}$ here (Evans et al., 1993).

In general, these parameters are fit numerically by an iterative algorithm that maximizes some goodness of fit (GOF) metric between the data $(\mathbf{y})$ and fitted response $\left(g\left(\mathbf{t}, p_{1}, p_{2}, p_{3}\right)\right)$. However, this GOF metric can have local maxima, particularly with noisy data for some participants and ROIs, and particularly when some parameters (such as onset latency and dispersion) have correlated effects on the fitted response. To accommodate this, we regularized the problem by imposing Gaussian priors on the parameters and on the noise, corresponding to a variational Bayesian approach that can be solved by maximizing a free-energy metric that approximates the model evidence (probability of the data given the model; Friston et al., 2007). In this case, $\mathbf{e}$ is assumed to be drawn from a zeromean Gaussian distribution with variance $p_{0}$. The prior mean of the onset latency $\left(p_{2}\right)$ was $0 \mathrm{~s}$, the prior mean of the dispersion factor $\left(p_{3}\right)$ was 1 , and the prior mean of the amplitude $\left(p_{1}\right)$ was set to the mean peak response over all regions, conditions, and participants $(0.76 \%$ signal change). The variance of each of these four priors for parameters $p_{0-3}$ was varied over a range [ 0.010 .10 .51510100$]$, and the maximal free-energy used to select the best of the resulting $7^{4}=2401$ models. This optimal model, which had prior variances of 10 for $p_{0}, 0.5$ for $p_{1}, 0.5$ for $p_{2}$, and 0.1 for $p_{3}$, was then used to estimate the posterior mean of the parameters reported in the main text.

Dynamic causal modeling. Dynamic casual modeling (DCM) was performed using version DCM10 in SPM8, using the same model described above (except that the inputs had duration of $2 \mathrm{~s}$, to allow sufficient sensitivity to modulation of connections (Henson et al., 2013). The volumes of interest were defined based on both anatomical and functional criteria. First, only voxels within the anatomically defined ROIs ( $\mathrm{PrC}$, $\mathrm{PhC}, \mathrm{HIPP}$ ) were considered. Second, within each ROI, we chose the 15 voxels with the strongest univariate effect sizes based on contrasts within a GLM: S-O(R) versus S-O(F) for $\operatorname{PrC}, \mathrm{O}-\mathrm{S}(\mathrm{R})$ versus $\mathrm{O}-\mathrm{S}(\mathrm{F})$ for $\mathrm{PhC}$, and $R$ versus $F$ (collapsed across object-cue and scene-cue trials) for HIPP. Note that this selection step merely served to identify the voxels most responsive within a given region and does not bias the subsequent DCM analysis to show any of the observed directionality effects. The top 15 voxels were identified for left and right hemisphere regions separately and then combined for the DCM analysis.

Bayesian model selection was performed using a random effects model, as described by Stephan et al. (2009). This allows estimation of the "exceedance probability," i.e., the extent to which each model is more likely than any other model tested to have generated the data from a randomly selected participant. The choice of models is described below.

\section{Results}

\section{Behavioral results}

During retrieval, the proportion of scene recall and forgot responses when cued with an object were 49.8 and $49.4 \%$, respectively (with no response given on $0.8 \%$ of the trials). The corresponding proportion for object recall when cued with a scene was 43.6 and $54.8 \%$, respectively (with no response given on the remaining $1.6 \%$ of the trials). Importantly, reaction times for recall responses did not differ statistically between object-cue and scene-cue trials (2.23 s vs $\left.2.16 \mathrm{~s} ; t_{(19)}=1.24, p=0.23\right)$. On randomly interspersed catch trials (where participants verbally described their memory after giving a recall keypress), the answer was correct on $98 \%$ of the trials when cued with an object and on $96 \%$ of the trials when cued with a scene, demonstrating that participants indeed recalled the correct target when indicating so. The average numbers of trials for our conditions of interest were 48 for O-S(R) (range 33-69), 47 for O-S(F) (range 26-62), 42 for $\mathrm{S}-\mathrm{O}(\mathrm{R})$ (range 25-61), and 52 for $\mathrm{S}-\mathrm{O}(\mathrm{F})$ (range 35-70).

\section{Functional segregation of $\mathrm{PrC}, \mathrm{PhC}$, and HIPP}

ROIs were hand-drawn individually for $\mathrm{PrC}, \mathrm{PhC}$, and HIPP (Fig. 2a), where PrC and PhC were defined as the anterior and posterior third of the parahippocampal gyrus, respectively (Staresina et al., 2011). Our first set of predictions (for functional segregation) concerned the involvement of different MTL regions as a function of retrieval success (recalled (R) vs forgotten (F)) and cue type (object (O) cue vs scene (S) cue). If the contri- 

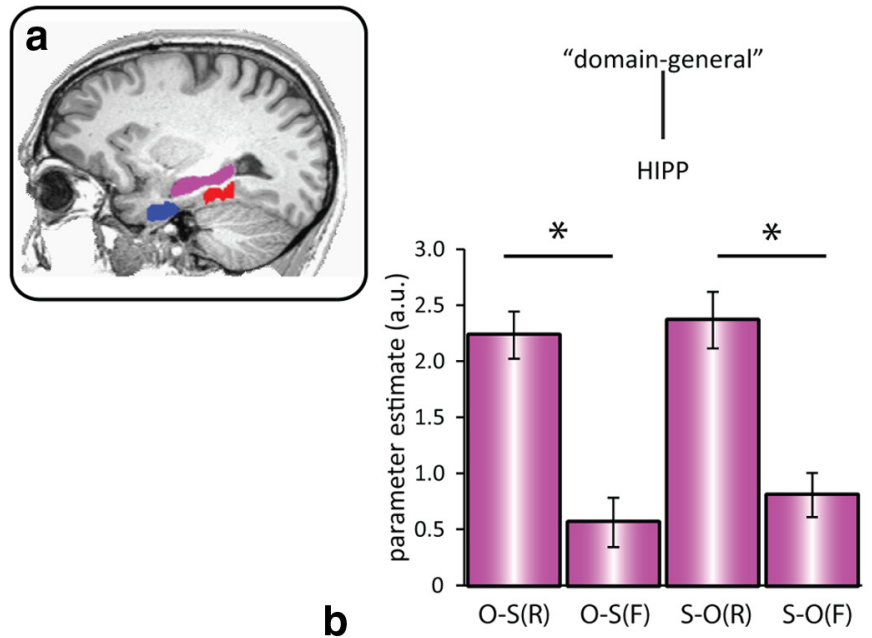

b

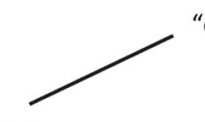

$\operatorname{PrC}$ object representations

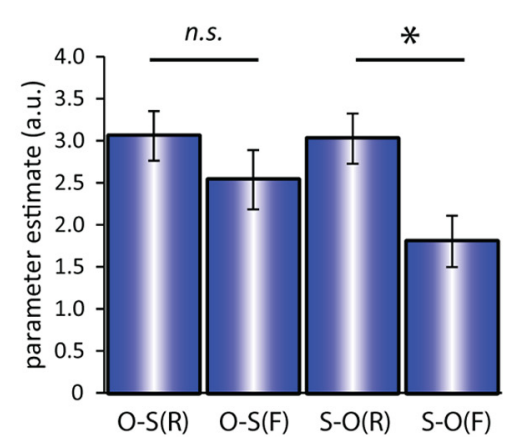

$\mathrm{PhC}$ scene representations

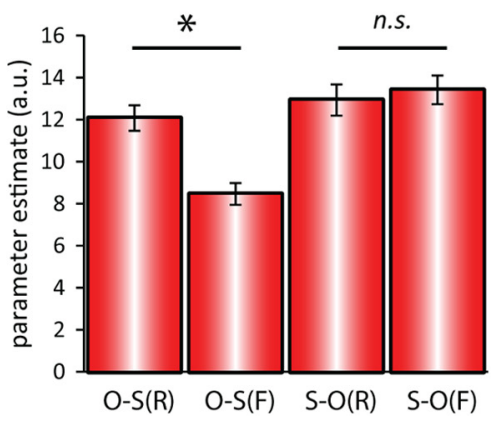

Figure 2. $\quad \boldsymbol{a}$, ROls, manually drawn for each participant (shown here for one example participant): $\operatorname{PrC}($ blue), $\operatorname{PhC}($ red), and HIPP (purple). $\boldsymbol{b}$, Mean ( \pm SEM) GLM parameter estimates in PrC (bottom left), PhC (bottom right), and HIPP (top) for successful versus unsuccessful recall as a function of cue and target stimulus $(N=20)$. Results show a three-way dissociation, where $\operatorname{PrC}$ and $\mathrm{PhC}$ are driven in a domain-specific fashion by object and scene representations, respectively (as the perceived cue or the retrieved target). Conversely, HIPP activation is driven in a domain-general fashion by success versus failure of recall, regardless of stimulus type. $0-\mathrm{S}(\mathrm{R})$ : object cue, scene target recalled; $0-\mathrm{S}(\mathrm{F})$ : object cue, scene target forgotten; $\mathrm{S}-\mathrm{O}(\mathrm{R})$ : scene cue, object target recalled; $\mathrm{S}-0$ (F): scene cue, object target forgotten. ${ }^{*} p<0.05$, two-tailed paired $t$ tests.

butions of $\mathrm{PrC}$ and $\mathrm{PhC}$ are domain specific, we would expect $\operatorname{PrC}$ to show a greater response during trials in which object information is represented, regardless of whether the object is perceived as the cue $(\mathrm{O}-\mathrm{S}(\mathrm{R})$ and $\mathrm{O}-\mathrm{S}(\mathrm{F}))$, or retrieved as the target after being cued with a scene image $(\mathrm{S}-\mathrm{O}(\mathrm{R}))$, compared with when no object information is perceived or retrieved $(\mathrm{S}-\mathrm{O}(\mathrm{F}))$. The same logic applies to PhC: an increased response would be expected whenever scene information is perceived $(\mathrm{S}-\mathrm{O}(\mathrm{R})$ and $\mathrm{S}-\mathrm{O}(\mathrm{F}))$, or retrieved from memory $(\mathrm{O}-\mathrm{S}(\mathrm{R}))$, compared with when no scene information is perceived or retrieved $(\mathrm{O}-\mathrm{S}(\mathrm{F}))$.

Using a conventional analysis of the parameter estimate for a canonical HRF derived from a GLM (see Materials and Methods), a repeated-measures ANOVA with the factors Region (PrC, PhC, HIPP), Cue Type (object, scene), and Memory (R, F) showed a highly significant three-way Region $\times$ Cue Type $\times$ Memory interaction $\left(F_{(1.41,26.87)}=110.33, p<0.001\right)$. Subsidiary repeated-measures ANOVAs conducted separately for each region showed a significant Cue Type $\times$ Memory interaction in $\operatorname{PrC}\left(F_{(1,19)}=8.39, p=0.009\right)$ and in $\operatorname{PhC}\left(F_{(1,19)}=94.86, p<\right.$ 0.001 ), but only a significant main effect of Memory in HIPP
$\left(F_{(1,19)}=95.17, p<0.001\right.$; Cue Type $\times$ Memory interaction, $F_{(1,19)}=0.31, p=$ $0.583)$. The pattern of significant pairwise differences is shown in Figure 2. In summary, $\operatorname{PrC}$ showed a significant memory effect (greater response to recalled than forgotten trials) for recalling objects, but not for recalling scenes; $\mathrm{PhC}$ showed a significant memory effect for recalling scenes, but not for recalling objects; and HIPP showed a significant memory effect for recalling both objects and scenes. This three-way interaction constitutes compelling evidence for functional segregation in the MTL: while PrC and PhC contributions are domain specific, driven by object and scene representations, respectively (either as the perceived cue or as the retrieved target), the contribution of HIPP is domain general and driven by success versus failure of associative recall. The same pattern of significant ROI results was obtained when allowing for latency differences (see below) by using instead as the dependent variable: (1) the percentage signal change from the peak time point of each region and condition or (2) the amplitude parameter from nonlinear fitting of the HRF.

\section{Latencies of evoked responses within $\mathrm{PrC}$ and $\mathrm{PhC}$}

If $\mathrm{PrC}$ and $\mathrm{PhC}$ provide domain-specific contributions, their relative engagement over time would be expected to vary as a function of the cue-target relationship. For instance, if $\mathrm{PrC}$ holds object representations, engagement of this region should occur earlier when an object serves as the cue than when an object is successfully retrieved as the target, assuming that information must undergo additional processing stages when retrieved from memory relative to being perceived in the environment. Likewise, engagement of $\mathrm{PhC}$ should occur earlier when a scene serves as the cue than when a scene is the successfully retrieved target. Correspondingly, we predicted an earlier response for $\mathrm{O}-\mathrm{S}(\mathrm{R})$ relative to $\mathrm{S}-\mathrm{O}(\mathrm{R})$ in $\mathrm{PrC}$, but an earlier response for $\mathrm{S}-\mathrm{O}(\mathrm{R})$ relative to $\mathrm{O}-\mathrm{S}(\mathrm{R})$ in $\mathrm{PhC}$, reflecting a reversal of the relative temporal ordering of conditions across regions.

Evidence for this prediction was apparent when plotting the trial-averaged time courses of the evoked BOLD response every $1 \mathrm{~s}$ (Fig. 3): while the greatest BOLD response in PrC occurred during the fifth TR for the O-S(R) condition (when averaging responses across participants), the greatest mean response for the $\mathrm{S}-\mathrm{O}(\mathrm{R})$ condition occurred later, in the sixth TR (Fig. 3a). The opposite pattern can be seen in $\mathrm{PhC}$ (Fig. 3b). To assess this statistically, we used nonlinear fitting of an HRF that was explicitly parameterized by its amplitude, onset latency, and dispersion (see Materials and Methods), and compared the onset latency estimates across conditions and regions. In $\mathrm{PrC}$, the average onset latency (relative to the stimulus onset at $0 \mathrm{~s}$ ) was $0.38 \mathrm{~s}$ for O-S(R) and $0.52 \mathrm{~s}$ for $\mathrm{S}-\mathrm{O}(\mathrm{R})$. In $\mathrm{PhC}$, on the other hand, the average 
onset latency was $0.65 \mathrm{~s}$ for $\mathrm{O}-\mathrm{S}(\mathrm{R})$ and $0.23 \mathrm{~s}$ for $\mathrm{S}-\mathrm{O}(\mathrm{R})$ (Fig. $3 c$ ). In a repeatedmeasures ANOVA with the factors Region (PrC, $\mathrm{PhC}$ ) and Cue Type (object, scene), the onset latencies showed a significant cross-over interaction $\left(F_{(1,19)}=15.23\right.$, $p=0.001)$. Importantly, no such difference in onset latencies was seen for the corresponding "forgot" trials $\left(F_{(1,19)}=\right.$ $1.98, p=0.175)$, and there was a significant three-way interaction between Region, Cue Type, and Memory $\left(F_{(1,19)}=\right.$ $10.48, p=0.004)$, suggesting that the delayed response indeed reflected the retrieval of the associated target, rather than general stimulus-related properties. There was no significant difference in HIPP between onset latency parameters for the $\mathrm{O}-\mathrm{S}(\mathrm{R})$ and $\mathrm{S}-\mathrm{O}(\mathrm{R})$ conditions $\left(t_{(19)}=1.40, p=0.177\right)$.

Interestingly, there was no significant Region $\times$ Cue Type interaction for the dispersion parameter $\left(F_{(1,19)}=0.65, p=\right.$ 0.431 ), which suggests that the above interaction between Region and Cue Type on BOLD onset latency reflected a true difference in onset of neural activity, rather than a difference in the duration of that activity. This is important because a difference in (peak) latency of a BOLD response can also arise if there is a difference in duration, rather than onset, of underlying neural activity (Henson et al., 2013). More specifically, under a simple convolution model of the BOLD response, an increase in the duration of neural activity will produce a more dispersed BOLD response, with an increase in BOLD amplitude and peak latency. However, this possibility is captured by our inclusion of an explicit dispersion parameter. In sum, although both $\mathrm{PrC}$ and $\mathrm{PhC}$ showed similar response amplitudes for the $\mathrm{O}-\mathrm{S}(\mathrm{R})$ and $\mathrm{S}-\mathrm{O}(\mathrm{R})$ conditions, time-resolved $\mathrm{BOLD}$ analysis revealed a temporal dissociation within and across these regions: The $\mathrm{PrC}$ response preceded the $\mathrm{PhC}$ response when an object cue elicited successful recall of a scene target (O-S(R) trials), but the $\mathrm{PhC}$ response preceded the $\mathrm{PrC}$ response when a scene cue elicited successful recall of an object target (S-O $(R)$ trials). This reversible temporal order across the MTL cortex suggests that information flows from cue to target region during successful recall.

\section{Dynamic interactions across PrC, PhC, and HIPP}

While the previous results are suggestive of a signaling cascade from the region representing the cue to the region representing the target, it is still unclear (1) whether there is a causal relationship between the two regions, in the sense that successful recall is driven by increased effective connectivity from the region representing the cue to the region representing the target, and (2) whether this directional flow encompasses HIPP, as suggested by our BOLD amplitude results (Fig. 2). To address these questions, we used DCM (Friston et al., 2003). Briefly, this method is based on simulating a dynamic model of neural activity within each region as a function of its connectivity to other regions, together with a region-specific hemodynamic model that maps such neural activity to the dependent variable, i.e., BOLD time series. Different sets of connections (networks) correspond to different models, and these models can be compared in terms of their Bayesian model evidence. Models are defined by (1) the location of driving inputs (here, the cues) to one or more regions, (2) the presence and direction of connections between regions (intrinsic connectivity), and (3) the connections between regions that are modulated by condition (here, successful vs unsuccessful cued recall). We grounded the basic network architecture (inputs and intrinsic connectivity) in primate anatomy (Suzuki and Amaral, 1994), in that the driving object and scene cue inputs entered the MTL via $\mathrm{PrC}$ and $\mathrm{PhC}$, respectively, and all regions were fully, reciprocally connected. The key question was then which of these intrinsic connections was modulated by success vs failure of cued recall.

To address the first question of causal information flow from cue region to target region, we directly compared two types of models (Fig. 4A). In model $M+$, recall success modulated the effective connectivity from the cue region to the target region, i.e., from $\mathrm{PrC}$ to $\mathrm{PhC}$ for $\mathrm{O}-\mathrm{S}$ trials, and from $\mathrm{PhC}$ to $\mathrm{PrC}$ for $\mathrm{S}-\mathrm{O}$ trials. This was contrasted with model $M-$, where recall success modulated the effective connectivity in the reverse direction, i.e., from the target region to the cue region (from $\mathrm{PhC}$ to $\mathrm{PrC}$ for O-S trials, and from $\mathrm{PrC}$ to $\mathrm{PhC}$ for S-O trials). Results showed com- 
A

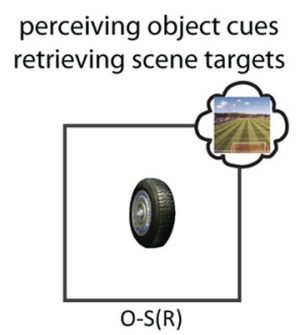

perceiving scene cues retrieving object targets

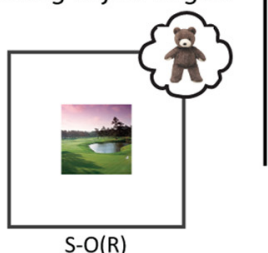

modulation of

connectivity by recall success

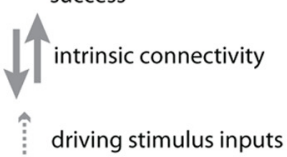

" $M-$ ": modulatory input from target- to cue region

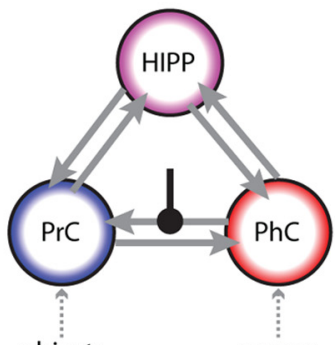

objects scenes

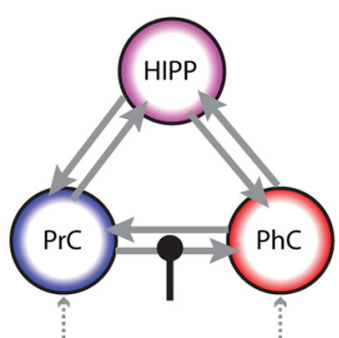

objects

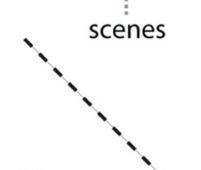

B

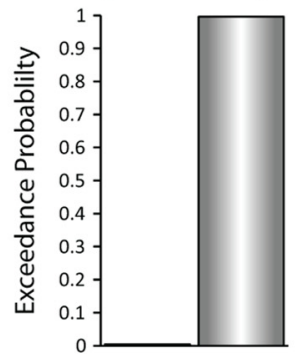

"M+": modulatory input

from cue- to target region
"M++": modulatory input
from cue- to target region
via HIPP

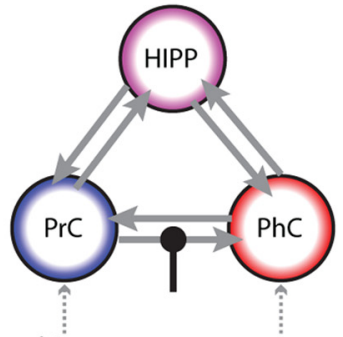

scenes

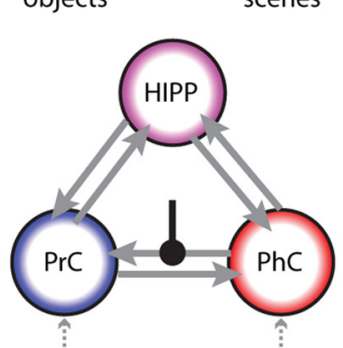

objects
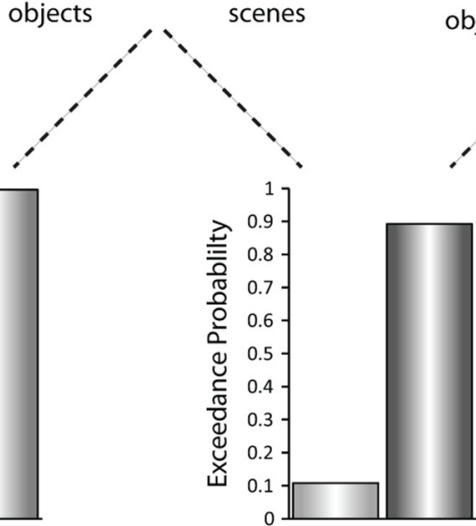

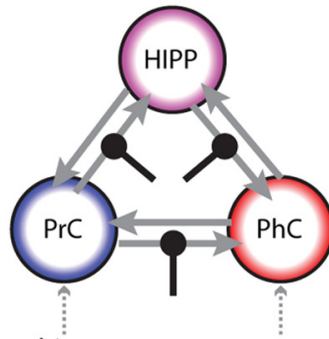

objects

scenes

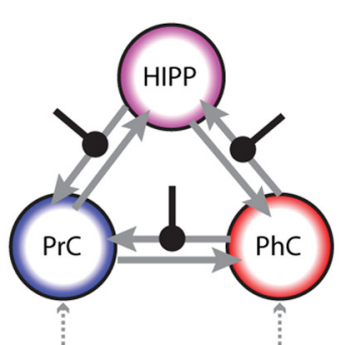

objects

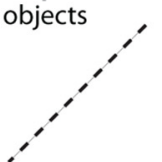

scenes

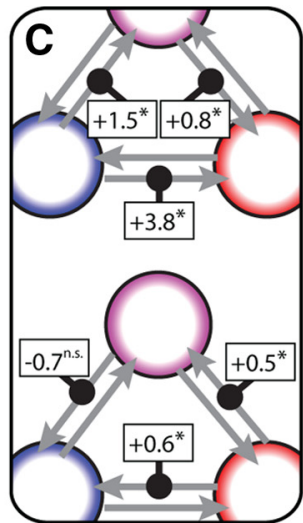

Figure 4. Causal network dynamics (DCM). A, Different models of information flow across the MTL for 0 -S trials (top) and S-0 trials (bottom). Models share the same network architecture of driving input and full intrinsic connectivity (gray arrows), but differ with regard to the connections modulated by recall success (black lines). Models $M-$ and $M+$ only encompass modulatory connections between MTL cortical regions and differ in their directional flow, from target region to cue region $(M-)$ versus from cue region to target region $(M+)$. Model $M++$ tested whether additional modulation of a hippocampal route improved the model evidence. $B$, Direct Bayesian model comparisons $(N=20)$, showing clear evidence in favor of a cue-to-target connection modulated by recall success (left) with an additional boost of the model evidence after inclusion of a modulated hippocampal route too (right). C, DCM connection strengths from a full model in which modulation was allowed of every connection. Top, 0 -S trials; bottom, $\mathrm{S}-0$ trials. Associated numbers are the sum of intrinsic and modulatory parameters, and asterisks indicate numbers significantly greater than zero, ${ }^{*} p<0.05$, one-tailed.

pelling evidence in favor of $M+$, with an exceedance probability of 0.996 (Fig. 4B, left).

While this result corroborates the notion of directed information flow from cue to target region that was suggested by the BOLD latency analysis (Fig. 3), it still leaves open whether successful recall encompasses information flow via the hippocampus. We thus devised a third model, which again had the same network architecture as $M+$, but additionally allowed modulation of connections from the cue region to the hippocampus and from the hippocampus to the target region $(M++)$. Results showed that inclusion of a hippocampal route strongly increased the model evidence (exceedance probability of 0.892 in a direct comparison; Fig. $4 B$, right). Note also that model $M++$ outperformed all other models in a larger model space that systematically varied all modulatory connections while holding driving inputs and intrinsic connectivity constant, confirming that this is the optimal model among these multiple alternatives. Last, we compared model $M++$ (in which the flow of information varies flexibly as a function of the cue and target stimulus type) with two alternative models, one in which the connectivity from $\mathrm{PrC}$ toward $\mathrm{PhC}$ was modulated by recall success for both O-S and S-O trials, and one in which the connectivity from $\mathrm{PhC}$ toward $\mathrm{PrC}$ was modulated by recall success for both O-S and S-O trials. In other words, the latter two models assumed that information would always flow in a particular direction, regardless of the cuetarget relationship. Again, the exceedance probabilities of those three models strongly favored the flexible bidirectional model $M++(0.67$ vs 0.15 and 0.18 , respectively).

As a final test, we fit a fourth, "full" model in which every connection to and from each region was modulated by recall success, and tested whether the coupling parameters were indeed reversible as a function of the cue-target relationship (O-S vs S-O trials). In a repeated-measures ANOVA on the modulatory coupling parameters, we included the factors Cue Type (object, 
scene) and Direction (from PrC toward $\mathrm{PhC}$ vs from $\mathrm{PhC}$ toward $\mathrm{PrC}$, averaging across PrC-PhC, PrC-HIPP, and PhC-HIPP connections). Critically, we observed a significant Cue Type $\times$ Direction interaction $\left(F_{(1,19)}=37.92, p<0.001\right)$, due to a significant increase in effective connectivity from $\operatorname{PrC}$ toward $\mathrm{PhC}$ (compared with $\mathrm{PhC}$ toward $\mathrm{PrC}$ ) during $\mathrm{O}-\mathrm{S}(\mathrm{R})$ trials $\left(t_{(19)}=5.25, p<0.001\right)$, but a significant increase in effective connectivity from $\mathrm{PhC}$ toward $\mathrm{PrC}$ (compared with $\mathrm{PrC}$ toward $\mathrm{PhC})$ during S-O $(\mathrm{R})$ trials $\left(t_{(19)}=4.08, p<0.001\right)$. Indeed, when testing the individual connection strengths during successful recall (the sum of the intrinsic and modulatory connection parameters), five of the six modulations in the forward direction (from cue region toward target region) were significantly greater than zero (Fig. 4C). This included the output connection from HIPP to $\mathrm{PhC}$ during $\mathrm{O}-\mathrm{S}$ trials, though the output connection from HIPP to PrC during S-O trials did not significantly differ from zero (see Discussion).

To summarize our DCM analysis: following the observation of a reversal of relative response latencies across $\mathrm{PrC}$ and $\mathrm{PhC}$ (Fig. 3), we obtained further evidence for a causal dynamic relationship between $\mathrm{PrC}$ and $\mathrm{PhC}$, such that $\mathrm{PrC}$ drives activation in $\mathrm{PhC}$ when $\mathrm{PrC}$ represents the cue and $\mathrm{PhC}$ represents the target, but $\mathrm{PhC}$ drives $\mathrm{PrC}$ when the cue-target assignment is reversed. We then went on to demonstrate that this directional flow from cue toward target region is better captured by adding a further indirect route via the hippocampus. This was demonstrated both across models that differed in which connections were modulated by successful recall, and across coupling parameters within a fully modulated model. Relating back to the pattern of functional segregation (Fig. 2), these results suggest that the hippocampus flexibly links domain-specific representations in MTL cortex during successful recall.

\section{Discussion}

Ever since the hallmark case of patient H.M. (Scoville and Milner, 1957), whose episodic memory was devastated by a large lesion to his MTLs, memory research has primarily focused on teasing apart the contributions of different regions within the MTL (Cohen and Eichenbaum, 1993; Aggleton and Brown, 1999; Cohen et al., 1999; Norman and O'Reilly, 2003; Eichenbaum, 2004, 2007; Squire et al., 2004; Henson, 2005; Davachi, 2006; Diana et al., 2007; Mayes et al., 2007). However, controversy about the precise principles of functional segregation has hindered progress on the arguably more important question of how these regions dynamically interact to enable our rich and integrated episodic memories (functional integration). The question of functional integration is not unique to memory research; it is a fundamental challenge in neuroscience that emerges whenever specialized modules must be integrated to enable coherent perception, thought, and action (Zeki and Shipp, 1988; Edelman, 1993; Friston, 2002; Macaluso and Driver, 2005). In the current study, we first showed a pattern of functional segregation across MTL regions that supports recent neuroanatomically based accounts of MTL functions. Building on this division of labor, we then proceeded to assess how the separate contributions are dynamically integrated during successful recall.

\section{Functional segregation-three-way dissociation in the contributions of PrC, PhC, and HIPP}

As mentioned in the Introduction, recent efforts to capture the division of labor among MTL regions have emphasized the anatomical inputs and stimulus representations processed by these regions (Lee et al., 2005; Buffalo et al., 2006; Davachi, 2006; Diana et al., 2007; Graham et al., 2010; Staresina et al., 2011; Wixted and Squire, 2011; Liang et al., 2013). Regarding the MTL cortex, our current data provide strong support for this view (Fig. 2). Engagement of $\mathrm{PrC}$ and $\mathrm{PhC}$ was driven by processing of objects or scenes, respectively, regardless of whether their preferred stimuli were perceived as a cue, or retrieved as a target. Regarding the retrieval effects, it is interesting to note that despite the strong interaction of Cue Type $\times$ Memory in both regions (reflecting differential recall effects for each region's preferred stimulus type), there was a numerical trend in $\mathrm{PrC}$ toward a recall effect for scene targets. This pattern is reminiscent of an fMRI study that assessed MTL activation during encoding of objects and spatial locations (Buffalo et al., 2006) and found only spatial encoding effects in $\mathrm{PhC}$, but both object and spatial encoding effects in PrC (albeit stronger effects for objects). Moreover, a recent study using a cued recall paradigm in which objects were used as items and scenes were used as contexts (Hannula et al., 2013) found recall effects in $\mathrm{PhC}$ only when retrieving the scene context, but recall effects in PrC both when retrieving the object item and the scene context. Collectively, these results suggest that the assignment of PrC to object processing versus $\mathrm{PhC}$ to scene processing may not be perfectly symmetrical. One explanation might be that at conventional fMRI resolutions, PrC may include signal from the adjacent entorhinal cortex, which processes both spatial and nonspatial representations along its mediolateral gradient (Schultz et al., 2012). Higher resolution imaging would be needed to address this possibility. Another explanation (as suggested by Buffalo et al., 2006) might be that there is stronger anatomical input from $\mathrm{PhC}$ to $\mathrm{PrC}$ than vice versa (Suzuki and Amaral, 1994). The stronger direct connections from $\mathrm{PhC}$ to $\mathrm{PrC}$ than from $\mathrm{PrC}$ to $\mathrm{PhC}$ may also explain why modulation of output from HIPP to PrC in the DCM analysis did not reach significance for scene cues (see Results).

On a related note, it is worth considering that the relatively strict criterion for recall responses likely induced a fairly conservative response bias, such that forgot responses may include less confident target recall and/or different levels of stimulus familiarity. Likewise, in searching for the associated target, participants may continue to mentally generate and scan multiple exemplars from the target category during $F$ trials. While such transient stimulus representations are unlikely to achieve the same representational fidelity as successfully retrieved targets, they may still engage $\mathrm{PrC}$ and $\mathrm{PhC}$ to certain levels. This would explain the clear above-baseline activation levels of $\mathrm{S}-\mathrm{O}(\mathrm{F})$ and $\mathrm{O}-\mathrm{S}(\mathrm{F})$ trials in $\mathrm{PrC}$ and $\mathrm{PhC}$ (Fig. 2), respectively. However, the key finding with regard to $\mathrm{PrC}$ and $\mathrm{PhC}$ activation levels is their dissociation in supporting recall of object targets versus scene targets, respectively, consistent with a role of these regions in domain-specific retrieval.

Unlike in the MTL cortex, we observed no domain specificity in HIPP; rather, HIPP engagement reflected success versus failure of cued recall regardless of the stimulus-type being recalled. This is in agreement with the idea that HIPP contributions are domain general (Cohen and Eichenbaum, 1993; Eichenbaum, 2004; Davachi, 2006; Staresina and Davachi, 2008; Konkel and Cohen, 2009; Kumaran et al., 2012), and is again consistent with the multimodal array of anatomical inputs this region receives ( $\mathrm{Su}-$ zuki and Amaral, 1994; Lavenex and Amaral, 2000; van Strien et al., 2009). There is abundant evidence for the role of HIPP in associative binding/pattern completion (for reviews, see Cohen and Eichenbaum, 1993; Aggleton and Brown, 1999; Squire et al., 2004; Davachi, 2006; Eichenbaum et al., 2007; Mayes et al., 2007; Konkel and Cohen, 2009), but our data are the first to reveal how 
recall modulates the functional connectivity of HIPP with other MTL structures. This is arguably more direct evidence for a role of HIPP in pattern completion than has been furnished by previous activation analyses. To be explicit, while our results on functional segregation across the MTL corroborate and extend pervious findings, the novel aspect of the current study is that we build on these different contributions to ask how their dynamic interplay enables memory retrieval, as elaborated below.

\section{Network dynamics across the MTL: from functional segregation to integration}

Given the stimulus-specific contributions of $\mathrm{PrC}$ and $\mathrm{PhC}$, we first asked whether their engagement reflects different stages in the MTL signaling cascade during episodic retrieval. Specifically, we hypothesized that during $\mathrm{O}-\mathrm{S}(\mathrm{R})$ trials, $\mathrm{PrC}$ activation reflects processing of the object cue, whereas $\mathrm{PhC}$ activation reflects retrieval of the scene target. Similarly, during $\mathrm{S}-\mathrm{O}(\mathrm{R})$ trials, $\mathrm{PhC}$ activation should reflect processing of the scene cue, whereas $\mathrm{PrC}$ activation reflects retrieval of the object target. Given that the cue, by definition, precedes the recalled item in a cued-recall paradigm (and given that the response latency for recalled responses was $\sim 2 \mathrm{~s}$; see Results), one would expect these different functions to be expressed with different temporal profiles: engagement of the region representing the perceived cue should precede engagement of the region representing the retrieved target. As illustrated in Figure 3, the data show: during O-S(R) trials, the PrC BOLD response preceded the $\mathrm{PhC}$ response, whereas during $\mathrm{S}-\mathrm{O}(\mathrm{R})$ trials, the $\mathrm{PhC} \mathrm{BOLD}$ response preceded the PrC response.

Is the BOLD response sensitive enough to reveal temporal differences across conditions at such a short timescale? Despite the tacit assumption that the fMRI signal is proportional to neural firing rates, skepticism is warranted when interpreting BOLD time course effects (Friston et al., 2000; Heeger and Ress, 2002; Logothetis and Wandell, 2004). Therefore, any main effect of Region would be difficult to interpret due to potentially different neural-to-BOLD mappings across regions, and any main effect of Cue Type would be difficult to interpret due to potentially different dynamics earlier in object-processing-pathways versus sceneprocessing-pathways. Importantly, however, the cross-over interaction of Region $\times$ Cue Type we observed here (Fig. $3 c$ ) rules out any such region-specific or processing-pathway explanations. Furthermore, although it is difficult to infer backward from BOLD latency differences to underlying neural latency differences, our BOLD latency findings were obtained from nonlinear fitting of a model that included separate parametrization of onset delay, amplitude, and dispersion-allowing for more confident interpretation of differences in the BOLD onset latency parameter in terms of neural onset latency. Indeed, in a previous fMRI study, BOLD latency differences in PrC and HIPP across different memory retrieval conditions were directly confirmed by intracranial electroencephalography recordings (Staresina et al., 2012).

While the latency of the trial averaged-evoked BOLD response is suggestive of a directional interplay between $\mathrm{PrC}$ and $\mathrm{PhC}$ during successful cued recall, simple latency differences are only indirect evidence for a causal relationship between the region representing the cue stimulus and the region representing the target stimulus. Such causality is better inferred from temporal dependencies between regions across the whole fMRI time series, as in DCM. Furthermore, the latency analysis did not illuminate the putative role of HIPP as a pattern completer in this cue-target cascade (Marr, 1971; Teyler and DiScenna, 1986; Treves and Rolls, 1994; Norman and O'Reilly, 2003; Teyler and Rudy, 2007). Indeed, recent studies using direct electrophysiological record- ings in humans have shown results partly consistent with the notion of HIPP as the interface between cue and target: a source retrieval signal was shown to be initiated in HIPP in response to a preceding old/new signal in PrC (Staresina et al., 2012), and an increase in HIPP firing rates and gamma power was shown to precede free recall of target items (Sederberg et al., 2007; GelbardSagiv et al., 2008). However, due to restricted coverage of cortical sites in those studies, the network dynamics between HIPP and stimulus-specific MTL cortical regions ( $\mathrm{PrC}$ and $\mathrm{PhC}$ ) during successful recall has remained elusive. Here, we included HIPP in a DCM model to explicitly test for changes in effective connectivity between all three MTL regions during successful memory retrieval. First and foremost, our DCM results provided clear evidence in favor of the expected flow of information across the MTL cortex (Fig. 4), i.e., from $\mathrm{PrC}$ toward $\mathrm{PhC}$ when perceiving an object and retrieving an associated scene, and in the opposite direction (from $\mathrm{PhC}$ toward $\mathrm{PrC}$ ) when perceiving a scene and retrieving an associated object. Second, a model that additionally incorporated an additional indirect transmission route, (1) from the cortical region representing the cue to HIPP and (2) from HIPP to the cortical region representing the target, further outperformed the model in which only direct $\mathrm{PrC}-\mathrm{PhC}$ connections were modulated. Again, this directional flow including the hippocampal route was reversible as a function of the cue-target relationship on a given trial. These results are consistent with the notion that HIPP serves as the site of associative recall/pattern completion, and that different parts of the MTL cortex serve as stimulus-specific input and output modules.

\section{References}

Aggleton JP, Brown MW (1999) Episodic memory, amnesia, and the hippocampal-anterior thalamic axis. Behav Brain Sci 22:425-444; discussion 444-489. Medline

Brainard DH (1997) The Psychophysics Toolbox. Spat Vis 10:433-436. CrossRef Medline

Brett M, Anton JL, Valabregue R, Poline JB (2002) Region of interest analysis using an SPM toolbox. Neuroimage 16:S497.

Buffalo EA, Bellgowan PS, Martin A (2006) Distinct roles for medial temporal lobe structures in memory for objects and their locations. Learn Mem 13:638-643. CrossRef Medline

Burwell RD, Amaral DG (1998) Cortical afferents of the perirhinal, postrhinal, and entorhinal cortices of the rat. J Comp Neurol 398:179-205. CrossRef Medline

Cohen NJ, Eichenbaum HE (1993) Memory, amnesia, and the hippocampal system. Cambridge, MA: MIT.

Cohen NJ, Ryan J, Hunt C, Romine L, Wszalek T, Nash C (1999) Hippocampal system and declarative (relational) memory: summarizing the data from functional neuroimaging studies. Hippocampus 9:83-98. CrossRef Medline

Dale AM (1999) Optimal experimental design for event-related fMRI. Hum Brain Mapp 8:109-114. CrossRef Medline

Davachi L (2006) Item, context and relational episodic encoding in humans. Curr Opin Neurobiol 16:693-700. CrossRef Medline

Diana RA, Yonelinas AP, Ranganath C (2007) Imaging recollection and familiarity in the medial temporal lobe: a three-component model. Trends Cogn Sci 11:379-386. CrossRef Medline

Edelman GM (1993) Neural Darwinism: selection and reentrant signaling in higher brain function. Neuron 10:115-125. CrossRef Medline

Eichenbaum H (2004) Hippocampus: cognitive processes and neural representations that underlie declarative memory. Neuron 44:109-120. CrossRef Medline

Eichenbaum H, Yonelinas AP, Ranganath C (2007) The medial temporal lobe and recognition memory. Annu Rev Neurosci 30:123-152. CrossRef Medline

Evans M, Hastings N, Peacock B (1993) Statistical distributions (Ed 2). New York: Wiley.

Friston K (2002) Functional integration and inference in the brain. Prog Neurobiol 68:113-143. CrossRef Medline 
Friston KJ, Mechelli A, Turner R, Price CJ (2000) Nonlinear responses in fMRI: the Balloon model, Volterra kernels, and other hemodynamics. Neuroimage 12:466-477. CrossRef Medline

Friston KJ, Harrison L, Penny W (2003) Dynamic causal modelling. Neuroimage 19:1273-1302. CrossRef Medline

Friston K, Mattout J, Trujillo-Barreto N, Ashburner J, Penny W (2007) Variational free energy and the Laplace approximation. Neuroimage 34: 220-234. CrossRef Medline

Gelbard-Sagiv H, Mukamel R, Harel M, Malach R, Fried I (2008) Internally generated reactivation of single neurons in human hippocampus during free recall. Science 322:96-101. CrossRef Medline

Graham KS, Barense MD, Lee AC (2010) Going beyond LTM in the MTL: a synthesis of neuropsychological and neuroimaging findings on the role of the medial temporal lobe in memory and perception. Neuropsychologia 48:831-853. CrossRef Medline

Hannula DE, Libby LA, Yonelinas AP, Ranganath C (2013) Medial temporal lobe contributions to cued retrieval of items and contexts. Neuropsychologia pii: S0028-3932(13)00053-5. CrossRef Medline

Heeger DJ, Ress D (2002) What does fMRI tell us about neuronal activity? Nat Rev Neurosci 3:142-151. CrossRef Medline

Henson R (2005) A mini-review of fMRI studies of human medial temporal lobe activity associated with recognition memory. Q J Exp Psychol B 58:340-360. CrossRef Medline

Henson R, Wakeman D, Phillips C, Rowe J (2013) Effective connectivity between OFA and FFA during face perception: DCM of evoked MEG, EEG and fMRI responses. Hum Brain Mapp, in press.

Insausti R, Juottonen K, Soininen H, Insausti AM, Partanen K, Vainio P, Laakso MP, Pitkänen A (1998) MR volumetric analysis of the human entorhinal, perirhinal, and temporopolar cortices. AJNR Am J Neuroradiol 19:659-671. Medline

Konkel A, Cohen NJ (2009) Relational memory and the hippocampus: representations and methods. Front Neurosci 3:166-174. CrossRef Medline

Konkle T, Brady TF, Alvarez GA, Oliva A (2010) Scene memory is more detailed than you think the role of categories in visual long-term memory. Psychol Sci 21:1551-1556. CrossRef Medline

Kumaran D, Melo HL, Duzel E (2012) The emergence and representation of knowledge about social and nonsocial hierarchies. Neuron 76:653-666. CrossRef Medline

Lavenex P, Amaral DG (2000) Hippocampal-neocortical interaction: a hierarchy of associativity. Hippocampus 10:420-430. CrossRef Medline

Lee AC, Barense MD, Graham KS (2005) The contribution of the human medial temporal lobe to perception: bridging the gap between animal and human studies. Q J Exp Psychol B 58:300-325. CrossRef Medline

Liang JC, Wagner AD, Preston AR (2013) Content representation in the human medial temporal lobe. Cereb Cortex 23:80-96. CrossRef Medline

Logothetis NK, Wandell BA (2004) Interpreting the BOLD signal. Annu Rev Physiol 66:735-769. CrossRef Medline

Macaluso E, Driver J (2005) Multisensory spatial interactions: a window onto functional integration in the human brain. Trends Neurosci 28:264271. CrossRef Medline

Marr D (1971) Simple memory: a theory for archicortex. Philos Trans R Soc Lond B, Biol Sci 262:23-81. CrossRef Medline

Mayes AR, Holdstock JS, Isaac CL, Montaldi D, Grigor J, Gummer A, Cariga P, Downes JJ, Tsivilis D, Gaffan D, Gong Q, Norman KA (2004) Associative recognition in a patient with selective hippocampal lesions and relatively normal item recognition. Hippocampus 14:763-784. CrossRef Medline

Mayes A, Montaldi D, Migo E (2007) Associative memory and the medial temporal lobes. Trends Cogn Sci 11:126-135. CrossRef Medline

Norman KA, O'Reilly RC (2003) Modeling hippocampal and neocortical contributions to recognition memory: a complementary-learningsystems approach. Psychol Rev 110:611-646. CrossRef Medline

Pruessner JC, Köhler S, Crane J, Pruessner M, Lord C, Byrne A, Kabani N,
Collins DL, Evans AC (2002) Volumetry of temporopolar, perirhinal, entorhinal and parahippocampal cortex from high-resolution MR images: considering the variability of the collateral sulcus. Cereb Cortex 12:1342-1353. CrossRef Medline

Schultz H, Sommer T, Peters J (2012) Direct evidence for domain-sensitive functional subregions in human entorhinal cortex. J Neurosci 32:47164723. CrossRef Medline

Scoville WB, Milner B (1957) Loss of recent memory after bilateral hippocampal lesions. J Neurol Neurosurg Psychiatry 20:11-21. CrossRef Medline

Sederberg PB, Schulze-Bonhage A, Madsen JR, Bromfield EB, Litt B, Brandt A, Kahana MJ (2007) Gamma oscillations distinguish true from false memories. Psychol Sci 18:927-932. CrossRef Medline

Squire LR (1992) Memory and the hippocampus: a synthesis from findings with rats, monkeys, and humans. Psychol Rev 99:195-231. CrossRef Medline

Squire LR, Stark CE, Clark RE (2004) The medial temporal lobe. Annu Rev Neurosci 27:279-306. CrossRef Medline

Staresina BP, Davachi L (2006) Differential encoding mechanisms for subsequent associative recognition and free recall. J Neurosci 26:9162-9172. CrossRef Medline

Staresina BP, Davachi L (2008) Selective and shared contributions of the hippocampus and perirhinal cortex to episodic item and associative encoding. J Cogn Neurosci 20:1478-1489. CrossRef Medline

Staresina BP, Duncan KD, Davachi L (2011) Perirhinal and parahippocampal cortices differentially contribute to later recollection of object- and scene-related event details. J Neurosci 31:8739-8747. CrossRef Medline

Staresina BP, Fell J, Do Lam AT, Axmacher N, Henson RN (2012) Memory signals are temporally dissociated in and across human hippocampus and perirhinal cortex. Nat Neurosci 15:1167-1173. CrossRef Medline

Stark CE, Squire LR (2001) When zero is not zero: the problem of ambiguous baseline conditions in fMRI. Proc Natl Acad Sci U S A 98: 12760-12766. CrossRef Medline

Stephan KE, Penny WD, Daunizeau J, Moran RJ, Friston KJ (2009) Bayesian model selection for group studies. Neuroimage 46:1004-1017. CrossRef Medline

Suzuki WA, Amaral DG (1994) Perirhinal and parahippocampal cortices of the macaque monkey: cortical afferents. J Comp Neurol 350:497-533. CrossRef Medline

Teyler TJ, DiScenna P (1986) The hippocampal memory indexing theory. Behav Neurosci 100:147-154. CrossRef Medline

Teyler TJ, Rudy JW (2007) The hippocampal indexing theory and episodic memory: updating the index. Hippocampus 17:1158-1169. CrossRef Medline

Treves A, Rolls ET (1994) Computational analysis of the role of the hippocampus in memory. Hippocampus 4:374-391. CrossRef Medline

van Strien NM, Cappaert NL, Witter MP (2009) The anatomy of memory: an interactive overview of the parahippocampal-hippocampal network. Nat Rev Neurosci 10:272-282. CrossRef Medline

Vann SD, Tsivilis D, Denby CE, Quamme JR, Yonelinas AP, Aggleton JP, Montaldi D, Mayes AR (2009) Impaired recollection but spared familiarity in patients with extended hippocampal system damage revealed by 3 convergent methods. Proc Natl Acad Sci U S A 106:5442-5447. CrossRef Medline

Wixted JT, Squire LR (2011) The medial temporal lobe and the attributes of memory. Trends Cogn Sci 15:210-217. CrossRef Medline

Yonelinas AP, Kroll NE, Quamme JR, Lazzara MM, Sauvé MJ, Widaman KF, Knight RT (2002) Effects of extensive temporal lobe damage or mild hypoxia on recollection and familiarity. Nat Neurosci 5:1236-1241. CrossRef Medline

Zeki S, Shipp S (1988) The functional logic of cortical connections. Nature 335:311-317. CrossRef Medline 